\title{
Design of a multilayer on-chip inductor by computational electromagnetic modelling
}

\author{
Muneeswaran Dhamodaran, Subramani Jegadeesan, \\ Arunachalam Murugan
}

\begin{abstract}
This paper presents a design of typical multilayer on-chip inductor to determine the layout parameters of the desired inductance value of electromagnetic modeling. The inductance and quality factor of multilayer on-chip spiral inductors are determined by its layout parameters and technological parameters. These layout parameters must be optimized to obtain the maximum quality factor at the desired frequency of operation. An electromagnetic model with fewer assumptions than empirical equations and higher efficiency than full-field solvers would be welcome. So would facile comparisons of different inductor structures. This paper describes recent works on the electromagnetic modeling of on-chip inductor structures applied to the comparison of inductor geometries, including the traditional spiral inductor and a novel multilayer inductor. The electromagnetic modeling of the investigative model is presented. The modeling and simulation are implemented using the method of moments. To simulate the proposed algorithm, the EM Simulator software is used.
\end{abstract}

K e y w ords: inductor, electromagnetic modeling, quality factor, mutual inductance, method of moments

\section{Introduction}

The multilayer on-chip inductor plays a key role in the design of on-chip radio-frequency (RF) equipment. For circuits such as voltage-controlled oscillators and lownoise amplifiers, the values of the inductance and the Q-factor are essential for the design of spiral inductors. The performance of the spiral inductor depends on the number of turns, line width, spacing, pattern and shape, number of metal layers, oxide thickness and conductivity of substrate. With the appearance of $0.25 \mu \mathrm{m}, 0.18 \mu \mathrm{m}$, and recent $0.13 \mu \mathrm{m}$ CMOS technologies, a complete RF system operating in the gigahertz range can be integrated on silicon with a conventional CMOS process including both active and passive components, such as a wireless transceiver. The on-chip inductor also plays an important role in monolithic RF analog integrated circuits. It is widely used in low-noise amplifiers, mixers, and voltagecontrolled oscillators [1].

Obtaining high inductance or high $Q$ for RF circuits with on-chip spiral inductors, which occupy a large surface area, has been a problem. However, the trend has been to pursue better on-chip inductors, for the complete system in a single chip has obvious gains. The search is on for novel on-chip spiral, multilayer and 3-D inductors with large $L$, small area, low loss and high $Q$. This requires modeling the inductance characteristics of such structures accurately and efficiently for automated design [2]
Integrating inductors on chip greatly increases system integrity and reduces the packaging parasitic effects. However, because of their large chip area and significant leakage of magnetic energy, on-chip inductors also affect the performance of high-frequency integrated circuits through an electromagnetic coupling [3]. Therefore, it is of great importance to accurately characterize onchip inductors. Thus, in this study, the design of a typical multilayer on-chip inductor is presented along with its electromagnetic modeling so as to determine the layout parameters of the desired inductance value.

\section{Rectangular on-chip inductor design}

Conventional on-chip inductor design follows the pattern of metal spirals. The metal is typically chosen to be on the top layer in order to reduce the parasitic capacitance between the metal and the silicon substrate [4-9]. Figure 1 shows a rectangular on-chip inductor.

In order to model the inductance of the entire structure, a rectangular spiral is decomposed into segments, and the total impedance is the summation of the selfimpedance of each segment and the mutual impedance between each two of them. The decomposition of a rectangular spiral is illustrated in Fig. 2. $N$ is the number of spiral turns, and the total number of segments is $4 N$. A spiral inductor of width $8 \mu \mathrm{m}$ with four turns was used to lay this out, and the edge-to-edge spacing between parallel segments was $2 \mu \mathrm{m}$. The spiral was $11.7 \mu \mathrm{m}$ above

*Department of Electronics and Communication Engineering, M. Kumarasamy College of Engineering, Karur, Tamil Nadu, 639113, India, dhamodaranm@yahoo.com 


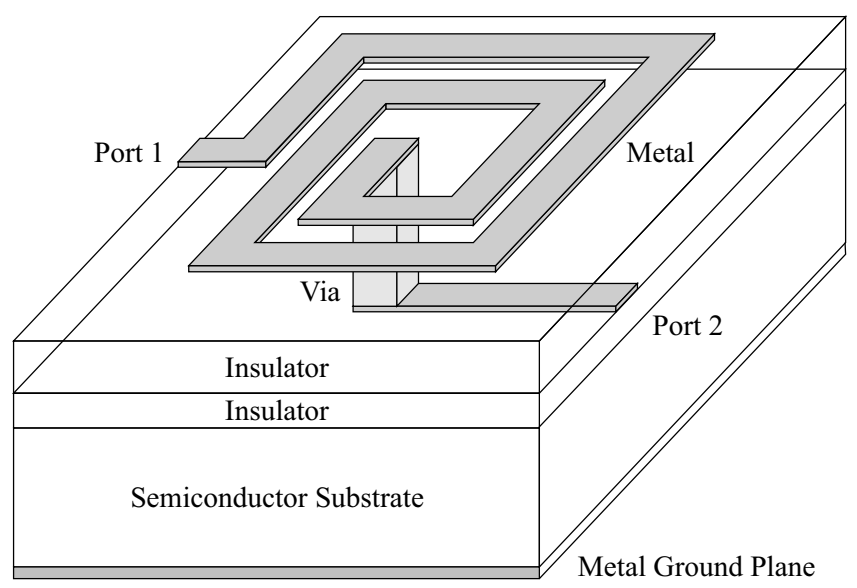

Fig. 1. On-chip rectangular spiral inductor

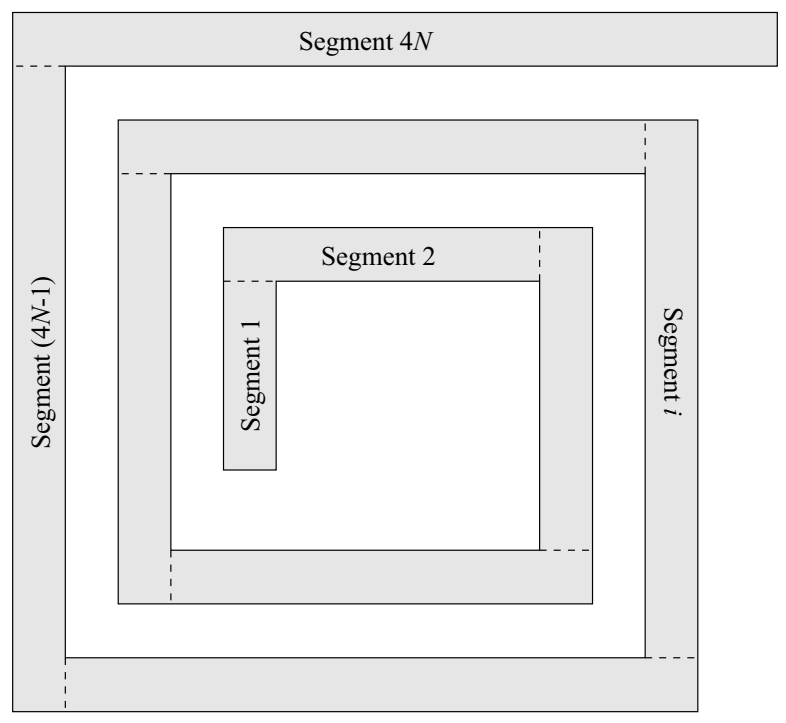

Fig. 2. Decomposition of an $N$-turn rectangular spiral

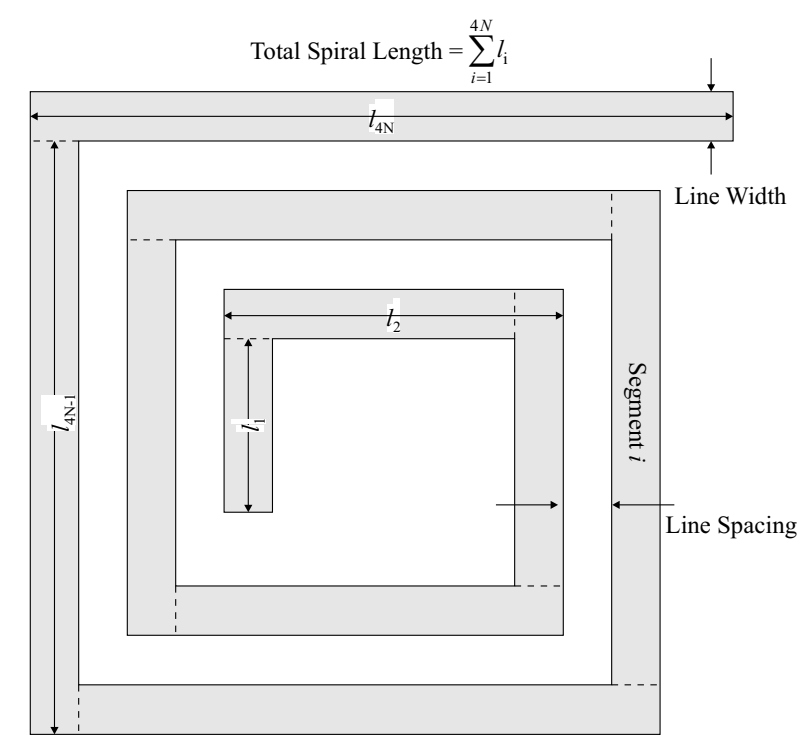

Fig. 3. Illustrations of spiral parameters a ground plane, and the conductor was assumed to have a thickness of $0.86 \mu \mathrm{m}$. The total length of the inductor was $2000 \mu \mathrm{m}$.

The total AC voltage drop across the two ports of the spiral is the summation of the voltage drop on each segment and is expressed as

$$
V=\sum_{i=1}^{4 N} V_{i}
$$

According to the inductance definition, the voltage drop on each segment is contributed by both the self-inductance and the mutual inductance between the segment and every other segment and is expressed as

$$
V_{i}=L_{i} \frac{\mathrm{d} I_{i}}{\mathrm{~d} t}+\sum_{\substack{j=1 \\ j \neq i}}^{4 N} L_{\mathrm{m}, i j} \frac{\mathrm{d} I_{j}}{\mathrm{~d} t}
$$

where $L_{i}$ is self-inductance, $L_{\mathrm{m}, \mathrm{ij}}$ is mutual inductance. Therefore, a matrix of voltage-current relationship can be established as

$$
\begin{aligned}
& {\left[\begin{array}{ccccc}
V_{1} & V_{2} & \ldots & V_{4 N}
\end{array}\right]^{\top}=} \\
& {\left[\begin{array}{cccc}
L_{11} & L_{m, 12} & \ldots & L_{m, 1-4 N} \\
L_{m, 21} & L_{22} & \ldots & L_{m, 2-4 N} \\
\vdots & \vdots & & \vdots \\
L_{m, 4 N-1} & L_{m, 4 N-2} & \ldots & L_{4 N-4 N}
\end{array}\right] \frac{\mathrm{d}}{\mathrm{d} t}\left[\begin{array}{c}
I_{1} \\
I_{2} \\
\vdots \\
I_{4 N}
\end{array}\right]}
\end{aligned}
$$

where the inductance matrix is

$$
\bar{L}=\left[\begin{array}{cccc}
L_{11} & L_{m, 12} & \ldots & L_{m, 1-4 N} \\
L_{m, 21} & L_{22} & \ldots & L_{m, 2-4 N} \\
\vdots & \vdots & & \vdots \\
L_{m, 4 N-1} & L_{m, 4 N-2} & \ldots & L_{4 N-4 N}
\end{array}\right] .
$$

In this matrix, the diagonal elements are the selfinductance of each segment, and the off-diagonal elements 

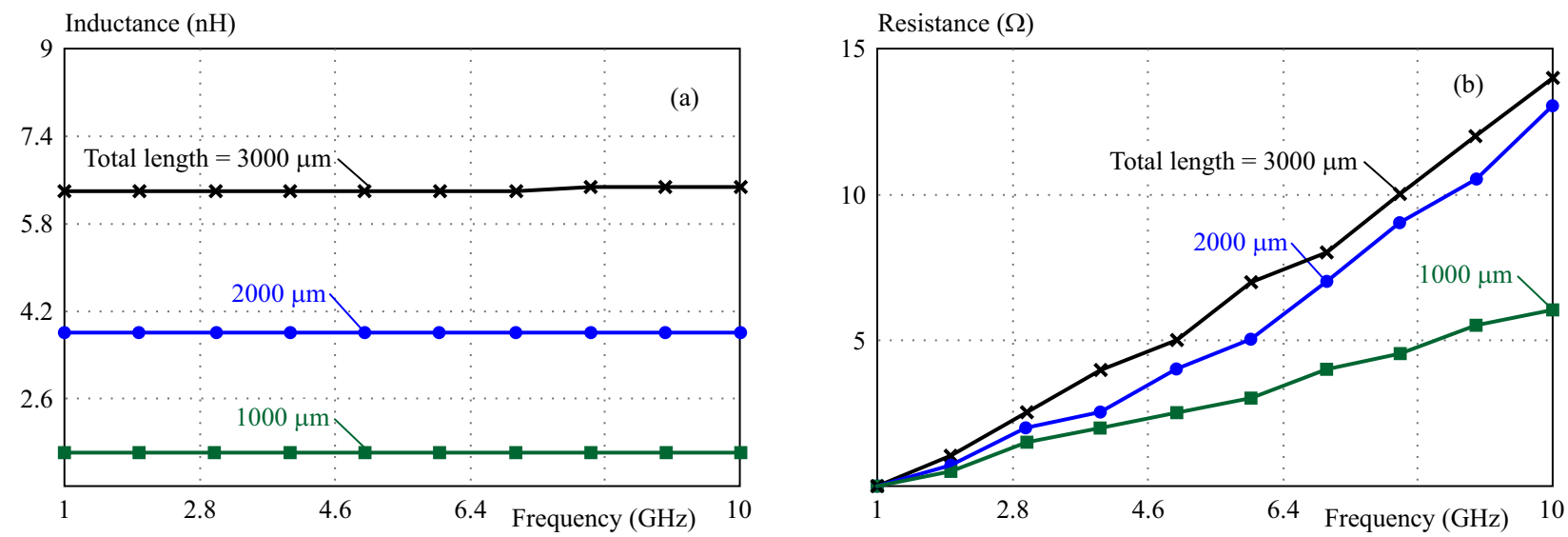

Fig. 4. Spiral inductor impedance with different total spiral lengths versus frequency
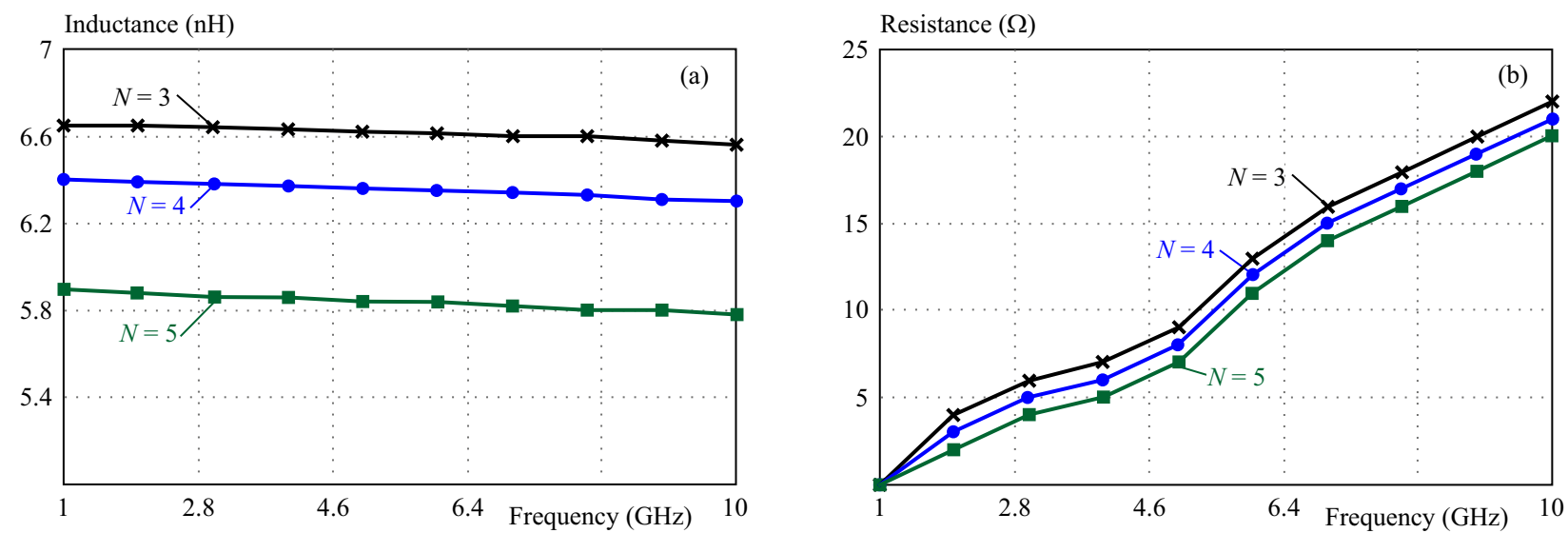

Fig. 5. Spiral inductor impedance with different number of turns versus frequency: (a) - inductance, (b) - resistance

are the mutual inductance between each two segments. Applying (1), the total inductance of the rectangular spiral is the summation of all the elements of the inductance matrix

$$
L_{\mathrm{tot}}=\sum_{i=1}^{4 N} \sum_{j=1}^{4 N} \bar{L}_{i j}
$$

Since the inductor is an on-chip component, the inductance of the spiral given by (5) is a complex inductance, which includes both inductance and resistance. The quality factor of an inductor is defined as

$$
Q=\frac{\omega L}{R}
$$

where $\omega$ is the angular frequency, $L$ is inductance and $R$ is resistance.

Quality factor of an on-chip spiral inductor increases with frequency and reaches a maximum value after which it decreases due to the ohmic loss in the series resistance and the loss in the substrate. To investigate the effect of varying width, turns, and spacing on quality factor when the inductance is constant. The quality factor improvement is important for multilayer inductor designs. In the inductor design smaller geometries provide high $Q$-factors compared to larger coils in the conventional method.

\section{Modelling of multilayer on-chip inductors}

The inductance of multilayer on-chip inductor depends on several factors such as total spiral length, number of turns, line spacing, line width, and substrate conductivity. These factors are illustrated in Fig. 3. The effects of these factors are studied by computer simulation. Figure 4 plots the impedance of a rectangular spiral with different total spiral lengths versus frequency, while keeping constant the number of turns, line spacing, line width, and substrate conductivity. For this, a $0.25 \mu \mathrm{m}$ CMOS process is chosen. The spiral is on the Metal 5 layers. The number of turns is set at 4 . The line width is set at $8 \mu \mathrm{m}$, and line spacing is set at $2 \mu \mathrm{m}$. The oxide thickness is $3.96 \mu \mathrm{m}$, and the substrate thickness is $250 \mu \mathrm{m}$. The metal conductivity is $3.70 \times 10^{7} \mathrm{~S} / \mathrm{m}$. The substrate doping level is assumed to be $10^{17} \mathrm{~cm}^{-3}$. The total length of the spiral chosen for three different models is $3000 \mu \mathrm{m}, 2000 \mu \mathrm{m}$ and $1000 \mu \mathrm{m}$.

It is seen from Fig. 4 that both the inductance and the resistance of the spiral are roughly proportional to the total spiral length. Therefore, increasing the total length not only increases the inductance but also gives rise to resistive loss and, thus, decreases the inductor quality factor. 

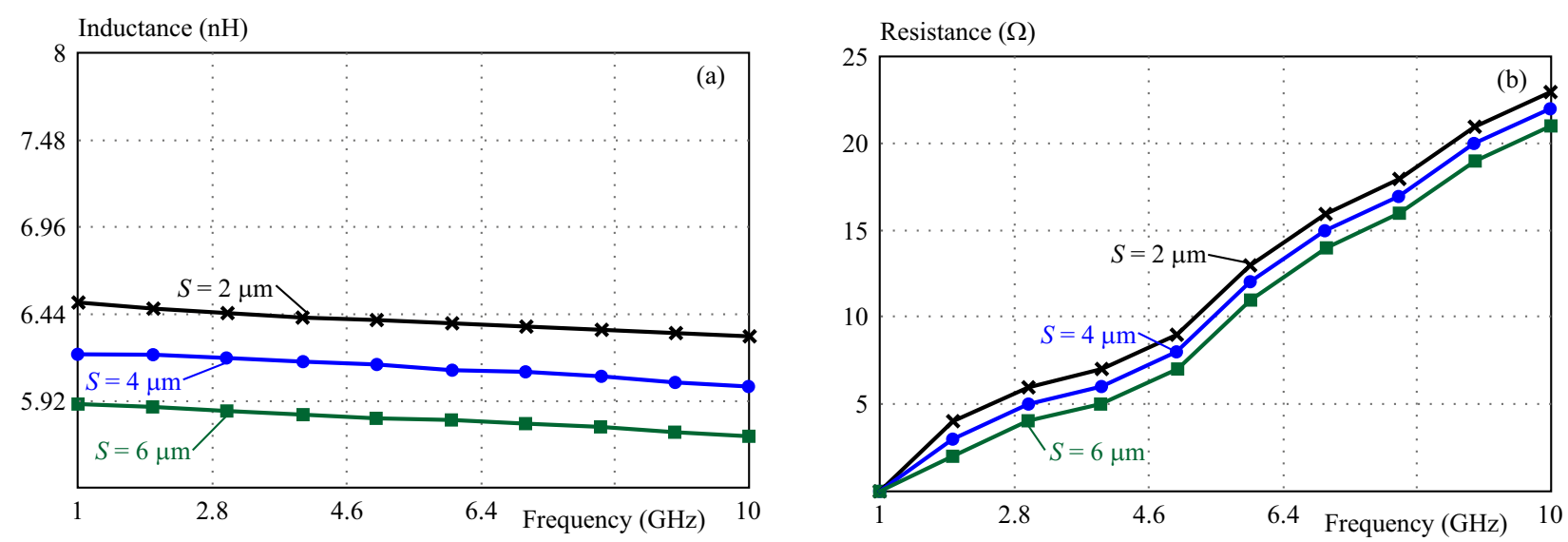

Fig. 6. Spiral inductor impedance with different line spacing versus frequency: (a) - inductance, (b) - resistance
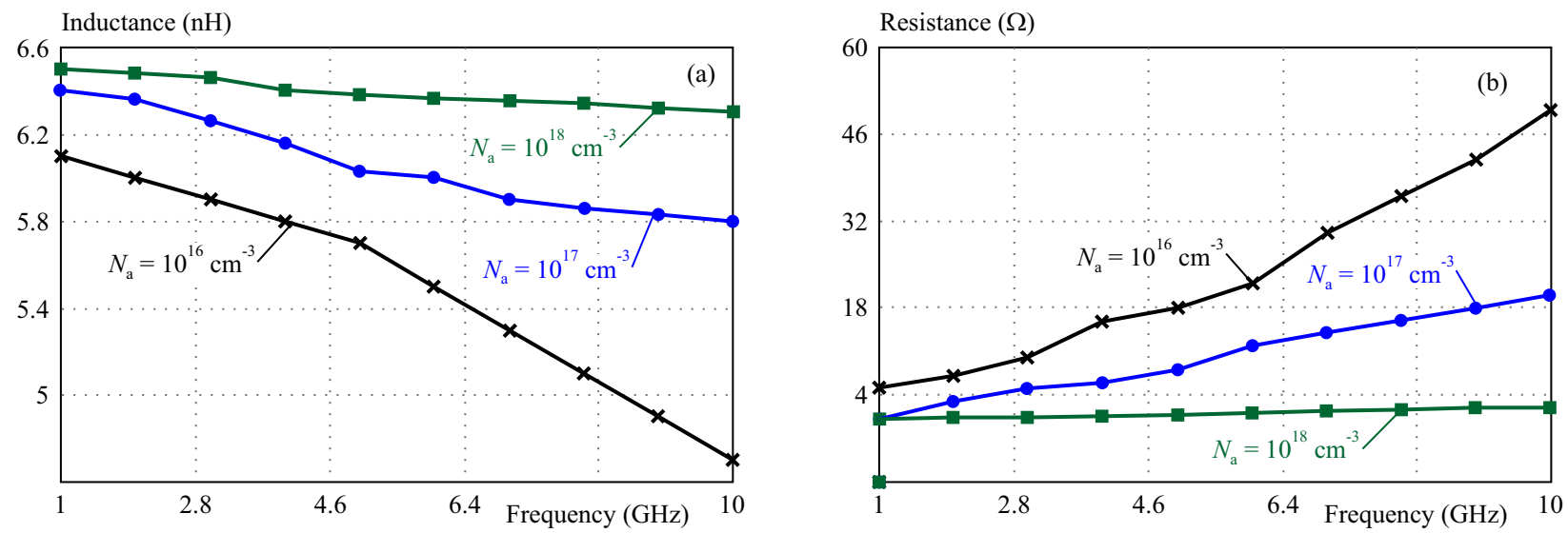

Fig. 7. Spiral inductor impedance with different substrate doping levels versus frequency: (a) - inductance, (b) - resistance

Figure 5 plots the impedance of a spiral inductor with different numbers of turns versus frequency, while keeping constant the total spiral length, line spacing, line width, and substrate conductivity. The spiral parameters are the same as the previous analysis, except that the total length is set at $3000 \mu \mathrm{m}$ and the number of turns varies.

It is evident from Fig. 5 that increasing the number of turns can effectively increase the spiral inductance as well as slightly decrease the resistance. This is because increasing the number of turns increases the total magnetic flux of the spiral [10].

Figure 6 plots the impedance of a spiral inductor with different line spacing versus frequency, while keeping constant the total spiral length, number of turns, line width, and substrate conductivity. The spiral parameters are the same as the previous analysis except that the line spacing varies.

It is seen from Fig. 6 that decreasing the line spacing is an effective way to increase spiral inductance without having much effect on the resistance. This is mainly because decreasing the line spacing increases the mutual inductance between the adjacent segments and, thus, the total inductance. The smallest line spacing is limited by the process design rule.

Figure 7 plots the impedance of a spiral inductor with different substrate doping levels versus frequency, while keeping constant the total spiral length, number of turns, line spacing, and line width. The spiral parameters are the same as the previous analysis except that the substrate conductivity varies.

It is seen from Fig. 7 that when the substrate doping level is low (around $N_{\mathrm{a}}=10^{18} \mathrm{~cm}^{-3}$ ), the inductance increases slightly at higher frequencies. This is because the skin effect in the substrate can be neglected. As a result of the skin effect, the current crowds on the edge of the conductor at higher frequencies. This implies a decreased spacing between the current elements in the adjacent segments and, therefore, an increased mutual inductance. However, if the substrate doping level is high, the spiral inductance decreases with increased frequencies because both the self-inductance of each segment and the mutual inductance between each two segments decreases at higher substrate doping levels. Similar analysis can be applied to spiral resistance, which increases at higher substrate conductivity.

\section{Multilayer on-chip inductors design}

The conventional spiral inductors are associated with several drawbacks. First, they occupy large chip areas. These include minimizing the noise figure of the amplifier, 


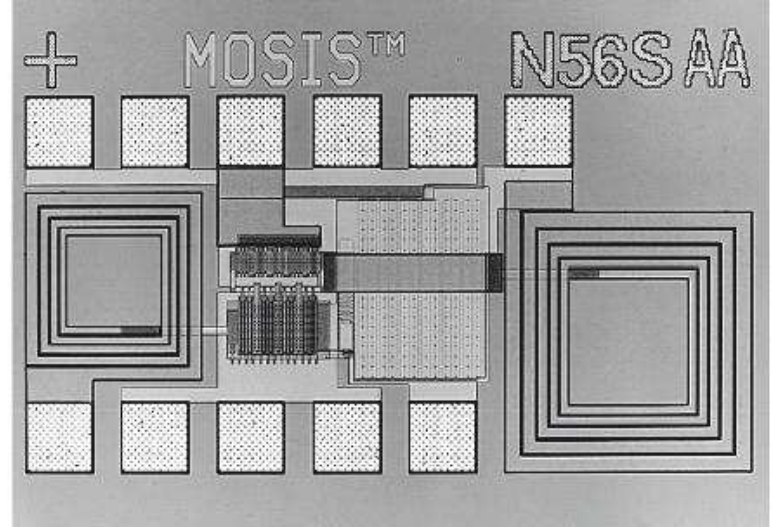

Fig. 8. Die photo of a $1.5 \mathrm{~V} 1.5 \mathrm{GHz}$ CMOS low-noise amplifier

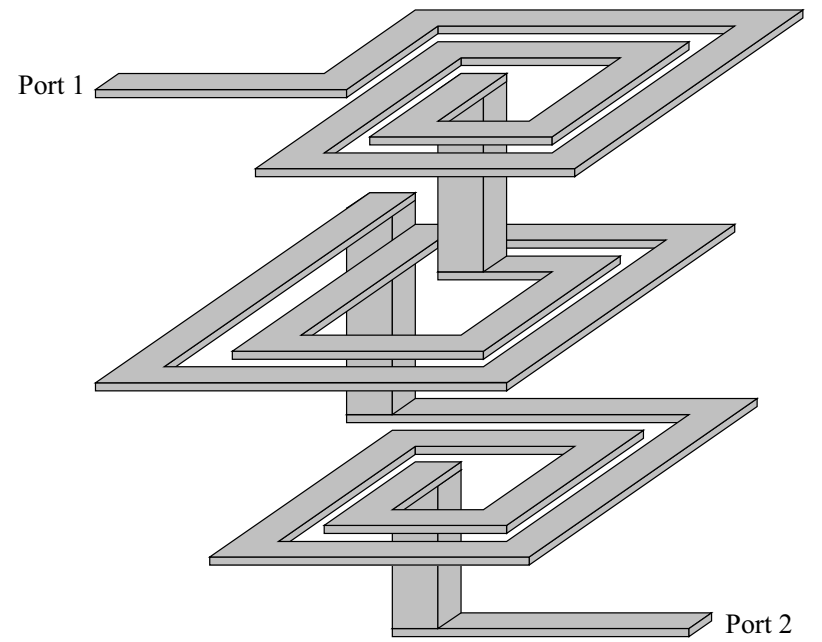

Fig. 9. Illustration of multilayer on-chip inductors
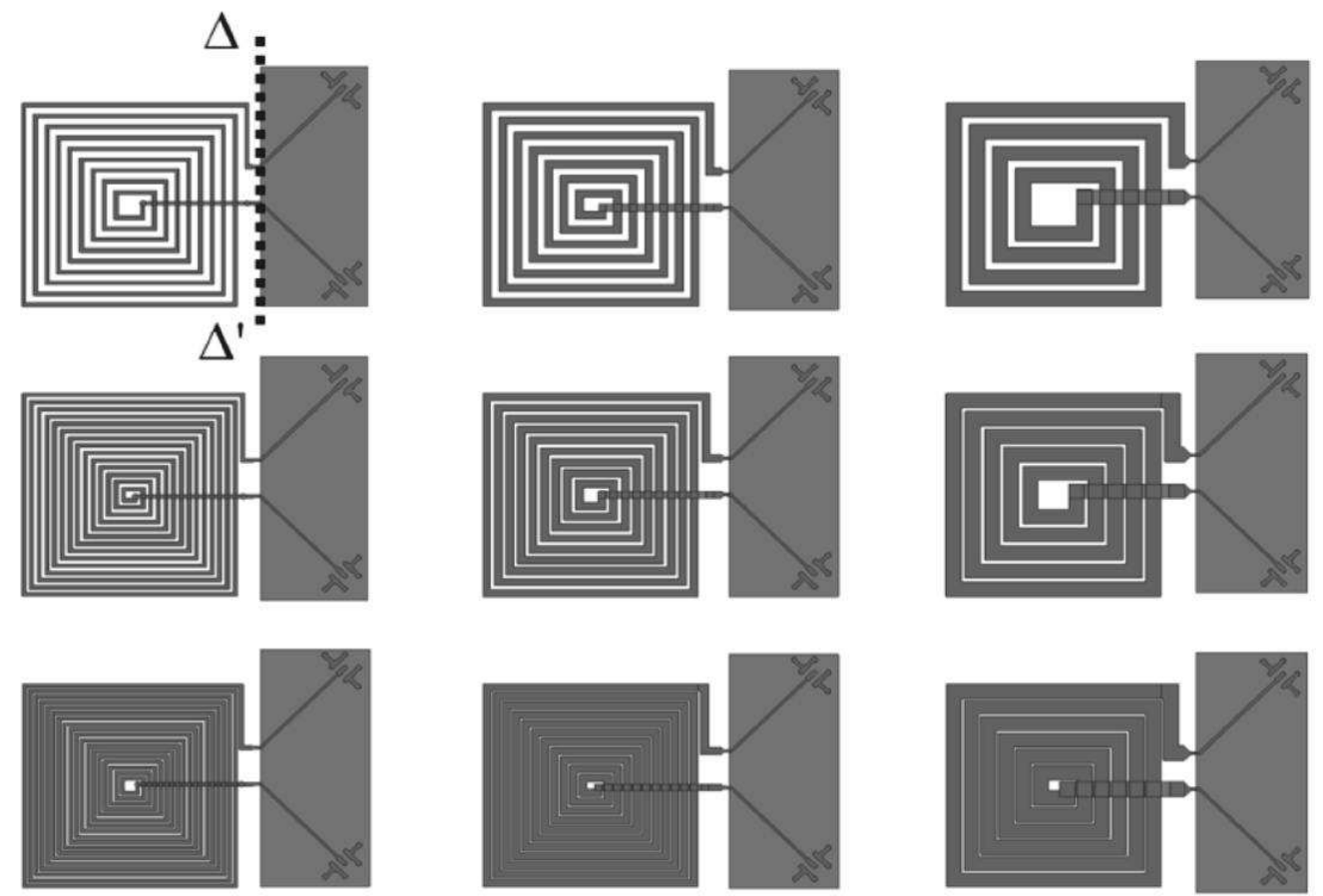

Fig. 10. The layout of multilayer inductor used for computer simulations

providing gain with sufficient linearity and providing a stable $50 \Omega$ input impedance to terminate an unknown length of transmission line. Figure 8 shows the die photo of a $1.5 \mathrm{GHz}$ CMOS low-noise amplifier [11], where two on-chip inductors take about two-thirds of the die area. The input pad is on the lower left corner of the die. The spiral on the left is a $4 \mathrm{nH}$ inductor, and the spiral on the right is a $7 \mathrm{nH}$ inductor that tunes the output of the first stage. The spirals are fabricated in metal [12].

It should be noted that it is hard to fit an inductor of more than $10 \mathrm{nH}$ on a chip. The inductance value is greatly limited for on-chip inductors. As a result, intermediate and low-frequency applications have to turn to off-chip alternatives. Second, because of the lossy semiconductor substrate on which an on-chip inductor is laid out, there is significant resistance as well as capacitive losses associated with on-chip inductors. Therefore, the quality factor of an on-chip inductor is much lower than their off-chip counterparts. The quality factor of on-chip inductors whose inductance is several $\mathrm{nH}$ will not go beyond ten in the lower $\mathrm{GHz}$ range.

Achieving high quality factor is the key goal of the on-chip inductor design. The inductor quality factor is directly related to the noise performance of RF circuits. An effective way to increase the quality factor of an on-chip inductor without decreasing the inductance is the implementation of multi-layer on-chip inductors. The concept of multi-layer inductors is illustrated in Fig. 9. Its advantage includes the use of multiple metal layers in the conventional CMOS processes. It consists of several stacked N-turn planar spirals. As seen from Fig. 9, all planar spirals have the same direction of current flow (either clock- 


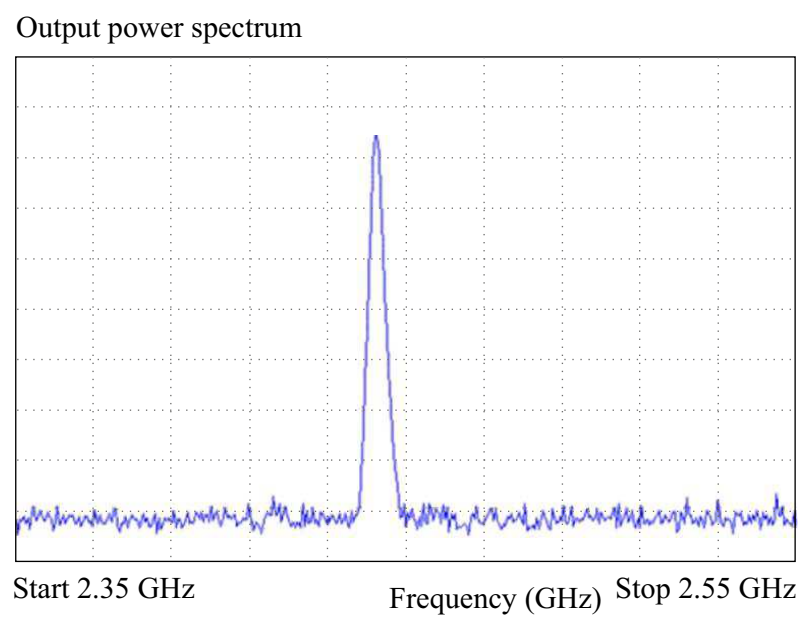

Fig. 11. Output power spectrum

Table 1. Numerical results for planar and proposed inductors

\begin{tabular}{lcc}
\hline Parameters & Planar & Proposed \\
\hline Resistance $(\Omega)$ & 3.117 & 3.117 \\
Inductor $(\mathrm{nH})$ & 1.5 & 0.452 \\
Length $(\mathrm{m})$ & 3000 & 1000 \\
Area $(\mathrm{m})$ & 14884 & 3186 \\
\hline
\end{tabular}

Table 2. Comparison of planar and proposed inductor $Q$-factors

\begin{tabular}{ccc}
\hline$f$ & \multicolumn{2}{c}{$Q$-factor } \\
$(\mathrm{GHz})$ & planar & proposed \\
\hline 1 & 1.4 & 1.8 \\
2 & 3.0 & 5.2 \\
3 & 6.0 & 8.0 \\
4 & 4.2 & 6.8 \\
5 & 2.8 & 4.4 \\
6 & 1.4 & 1.8 \\
\hline
\end{tabular}

wise or counterclockwise) to maximize the magnetic coupling between them. There are several publications on the experimental investigation of such 3D on-chip inductors [13]. The results of the electromagnetic simulations are verified by measurements of the multilayer spiral inductors. The inductors are processed on a different substrate layer. The different substrate has two $100 \mu \mathrm{m}$ thick dielectric layers and can be considered a good simulation of a flexible foil substrate.

Multiple inductors with variable track width and spacing are processed in the form of a matrix. Both variations are made simultaneously, thus accounting for unique multilayer spiral inductor designs, as shown in Fig. 10. This metal sheet acted as the ground plane. Based on the observations made on the results of the simulations, a multilayer spiral inductor can be designed. The design of the multilayer spiral inductor for on-chip RF applications is presented. An electrical model parameter is present.
The electromagnetic simulations are physically verified by measuring the multilayer spiral inductor designs.

The behavior of spiral inductors is analyzed. Resistive losses and quality factor are modeled by closed expressions that depend on geometrical parameters and the fabrication process. The inductance value is predicted within the limit against measured values for a set of spiral inductors. Our model can predict the frequency at which maximum $Q$ is obtained. This has been validated against measured data [14-16]. This methodology can be extended to other technologies by varying only the technological process parameters. Figure 11 shows the output spectrum of a carrier frequency range from $2.35 \mathrm{GHz}$ to $2.55 \mathrm{GHz}$. The output power is measured $-10.54 \mathrm{dBm}$ at $2.44 \mathrm{GHz}$.

As Table 1 shows, the proposed inductor saves the area cost. With the fixed resistance, it only uses $20 \%$ of the area occupied by conventional design. Thus, it is more compact and more economic for on-chip design. For future technology generations, as the number of metal levels and the distance from top metal to the substrate will increase dramatically. Even for current technology, the $Q$ factor may not be degraded.

\section{Results and discussion}

The process parameter variation response is observed in Fig. 4 to Fig. 7 for different total spiral length, different number of turns, different line spacing and different substrate doping, respectively, of same dimensions of inductor structure in three cases. Improving the quality factor is important for all inductor designs. When using an inductor in a circuit where the quality factor is important its resistance becomes an important factor. Any resistance will reduce the overall inductor $Q$ factor. An inductor can be considered in terms of its equivalent circuit.

Achieving high quality factor is the key goal of the on-chip inductor design. The inductor quality factor is directly related to the noise performance of RF circuits. An effective way to increase the quality factor of an on-chip 
inductor without decreasing the inductance is the implementation of multi-layer on-chip inductors. The quality factor of planar inductors will be higher than multilayer inductors.

The advantage of the proposed method becomes very clear when the design is compared with planer inductor. The comparison is given in the Tab. 2. The inductor has a peak quality factor of 8 under differential excitation and the frequency of $3 \mathrm{GHz}$. At $4 \mathrm{GHz}$ the quality factor is around 6.8. The structure is simulated using an EM simulator by defining all the process parameters. The structure was simulated from 1 to $7 \mathrm{GHz}$. From this proposed technique we can achieve a higher $Q$ factor.

\section{Conclusions}

In this study, an efficient technique for multilayer onchip spiral inductor has been developed and tested for many dimensions of the spiral inductor by changing the process parameter such as different total spiral length, different number of turns, different line spacing, and different substrate doping. This technique can achieve a higher $Q$ factor. The proposed multilayer on-chip spiral inductor is designed based on the fabrication rules of $180 \mathrm{~nm}$, which is a layout specific model. The on-chip area of these proposed inductors is very low when compared to planar inductor.

\section{REFERENCES}

[1] G. Haobijam and R. Paily, "Efficient Optimization of Integrated Spiral Inductor with Bounding of Layout Design Parameters", Analog Integrated Circuits Signal Processing, vol. 51, no. 3, pp. 131-140, 2007.

$[2]$ B. V. N. S. M. N. Deevi and N. B. Rao, "Multi-Layer On-Chip Inductor for $10-100 \mathrm{GHz}$ Frequency Applications", Electronics Letters, vol. 51, no. 3, pp. 270-272, 2015.

[3] S. J. Pan, L. W. Li, and W. Y. Yin, "Performance Trends of On-Chip Spiral Inductors for RFICs", Progress Electromagnetics Research PIER, vol, 45, pp. 123-151, 2004.

[4] M. Dhamodaran, R. Praveen Kumar and S. Jegadeesan, "OnChip Spiral Inductors On-Chip Spiral Transistors for Accurate Numerical Modeling", Journal of Magnetics, vol. 23, no. 1, pp. 50-54, 2018.

[5] C. P. Yue and S. S. Wong, "On-Chip Spiral Inductors with Patterned Ground Shields for Si-Based RF ICs", IEEE Journal of Solid-State Circuits, vol. 33, no. 5, pp. 743-752, 1998.

[6] C.-J. Chao, S.-C. Wong, C.-H. Kao, M.-J. Chen, L.-Y. Leu and K.-Y. Chiu, "Characterization Modeling of On-Chip Spiral Inductors for Si RFICs", IEEE Transactions on Semiconductor Manufacturing, vol. 15, no. 1, pp. 19-29, 2002.

[7] J. Chen and J.-J. Liou, "On-Chip Spiral Inductors for RF Applications: An Overview", Journal of Semiconductor Technology Science, vol. 4, pp. 149-169, 2004.

[8] N. A. Talwalkar, C. P. Yue and S. S. Wong, "Analysis and Synthesis of On-Chip Spiral Inductors", IEEE Transactions on Electron Devices, vol. 52, no. 2, pp. 176-182, 2005.

[9] N. D. Arora, K. V. Raol, R. Schumann, and L. M. Richardson, "Modeling Extraction of Interconnect Capacitances for Multilayer VLSI Circuits", IEEE Transactions on Computer-Aided
Design of Integrated Circuits systems, vol. 15, no. 1, pp. 58-67, 1996.

10] D. Rueter, "Induction Coil as a Non-Contacting Ultrasound Transmitter Detector: Modeling of Magnetic Fields for Improving the Performance", Ultrosonics, vol. 65, pp. 200-210, 2016.

[11] D. K. Shaeffer and T. H. Lee, "A $1.5 \mathrm{~V} 1.5 \mathrm{GHz}$ CMOS Low Noise Amplifier", IEEE Journal of Solid-State Circuits, vol. 32, no. 5, pp. 745-759, 1997.

[12] J. Gil and H. Shin, "Simple Wide-Band On-Chip Inductor Model for Silicon-Based RF ICs", IEEE Transactions on Microwave Theory Techniques vol 51, no, 9, pp, 2023-2028, 2003.

13] O. B. Kobe, J. Chuma, R. Jamisola Jr and M. Chose, "A review on quality factor enhanced on-chip microwave planar resonators", Engineering Science Technology an International Journal,, vol. 20, no. 2, pp. 460-466, 2017.

[14] Y. K. Koutsoyannopoulos and Y. Papananos, "Systematic analysis modeling of integrated inductors transformers in RF IC design", IEEE Transactions on Circuits Systems II: Analog Digital Signal Processing, vol. 47, no. 8, pp. 699-713, 2000.

[15] C. P. Yue and S. S. Wong, "Physical modelling of spiral inductors on silicon", IEEE Transactions on Electron Devices vol, 47, no, 3,, pp. 560 - 568, 2000.

16] E. F. Gutierrez-Frias, L. A. Garcia-Lugo, E. C. Becerra-Alvare J. J. Raygoza-Panduro, J. M. de la Rosa, E. B. Ortega-Rosales, "Methodology to improve the model of series inductance in CMOS integrated inductors", Journal of Electrical Engineering, vol. 69, no 3, pp. 250-254, 2018.

Received 6 June 2019

Muneeswaran Dhamodaran received the BE and ME degrees from Madurai Kamaraj University, Tamilnadu, India in 1993 and 1999, respectively, and the PhD degree from Anna University, India in 2016. He is currently working as a Professor in the Electronics and Communication Engineering Department at M. Kumarasamy College of Engineering, Karur, India, which is affiliated with Anna University, Chennai. He has been the author/coauthor of over 34 technical papers published in national and international Journals/Conference proceedings. His research interests include Computational Electromagnetics, Wireless Communication, Measurements, VLSI Design and EMI/EMC. He is a life member of IETE and Society of EMC Engineers.

Subramani Jegadeesan received the BE Degree in Electronics and Communication Engineering from Periyar University, Salem, India, in 2004 and the ME Degree in Communication Systems from Anna University, Coimbatore, India, in 2009. He received his $\mathrm{PhD}$ degree in the faculty of Information and Communication Engineering from Anna University, Chennai, in 2016. He is currently working as an Associate professor in the ECE Department, M.Kumarasamy College of Engineering, Karur, Tamilnadu, India. His main thirst research areas include energy management in wireless sensor networks, IoT, network and information security.

Arunachalam Murugan received the BE degree in Electronics and Communication Engineering from Bharathiyar University in 1990, the ME degree in Process Control and Instrumentation from Annamalai University in 2002 and the $\mathrm{PhD}$ degree in the faculty of Information and Communication Engineering from Anna University, Chennai, in 2019. He is currently working as an Associate professor in the ECE Department, M.Kumarasamy College of Engineering, Karur, Tamilnadu, India. His main thirst research areas include Machine Learning, Image processing, IoT, Network and Information Security. 